\title{
Measurement of the efficiency and time resolution of double-gap Resistive Plate Chambers
}

\author{
T.Moers and H.Tuchscherer a \\ III Physikalische Institut, RWTH Aachen, Germany \\ G.L.Bencze ${ }^{b}$, E.Radermacher, C.Seez ${ }^{c}$ and G.Wrochna ${ }^{d}$ \\ CERN, Geneva, Switzerland
}

C.Bacci e, F.Ceradini e, G.Ciapetti, F.Lacava, G.Margutti, A.Nisati, D.Orestano, E.Petrolo, L.Pontecorvo, A.Tusi, S.Veneziano, M.Verzocchi and L.Zanello

Dipartimento di Fisica dell'Universitd di Roma "La Sapienza" and INFN, Rome, Italy

R.Cardarelli, A.Di Ciaccio and R.Santonico

Dipartimento di Fisica dell'Università di Roma "Tor Vergata" and INFN, Rome, Italy

F.Szoncso, G.Walzel and C.-E.Wulz

Institut für Hochenergiephysik der Österreichischen Akademie der Wissenschaften, Vienna, Austria

\begin{abstract}
. ,
We describe a detector built by assembling together two Resistive Plate Chambers and read out by a single median electrode plane segmented in strips. We present results of measurements of the efficiency and of the time response as a function of the particle flux.
\end{abstract}
a) Now at University of Alabama, Tuscaloosa, USA.
b ) Visitor from Central Research Institute for Physics, KNKI, Budapest, Hungary
c) Visitor from Imperial College, London, U.K.
d) Visitor from Institute for Experimental Physics, Warsaw University, Warsaw, Poland
e) Dipartimento di Fisica della III Università di Roma, Rome, Italy 


\section{Introduction.}

We have already presented results of the performance of Resistive Plate Chambers, RPC, built with plates of phenolic polymers (bakelite) of different resistivities [1,2]. The measurements were done within the research and development programme of the RD5 experiment at CERN [3] to study the detection of large momentum muons in experiments at the Large Hadron Collider, LHC.

Experiments at the LHC [4] propose to build thousands of square meters of muon detectors made of drift chambers, to measure the muon trajectories with a precision of $\approx 100 \mu \mathrm{m}$, and fast hodoscopes finely segmented that provide a tracking trigger with a time resolution of few nanoseconds. A good time resolution is needed to uniquely identify the bunch crossing, that has a period of $25 \mathrm{~ns}$, at first level trigger. The use of RPC for the first level muon trigger was suggested [5] for their fast response and good time resolution, flexibility in segmentation, robustness and the low cost of production.

In the proposed experiments the muon detectors will be placed behind a thick calorimeter of 10-12 interaction lengths that absorb most of the showers produced by hadrons emitted with large transverse momenta. At a luminosity of $10^{34} \mathrm{~cm}^{-2} \mathrm{~s}^{-1}$ a charged particle flux of $(0.1-1) \mathrm{Hz} / \mathrm{cm}^{2}$ is expected in the barrel region, $|\eta| \leq 1.2$, increasing up to $\approx 100 \mathrm{~Hz} / \mathrm{cm}^{2}$ at $|\eta|=2$. Secondary interactions in the beam pipe and in the forward regions of the experiments will produce a much larger flux, $\left(10^{4}-10^{5}\right)$ $\mathrm{Hz} / \mathrm{cm}^{2}$, of very low energy neutrons and photons almost uniform over the surface of the detectors [6]. The sensitivity of RPC to low energy neutrons and photons is under study, preliminary results show that it is of $10^{-3}-10^{-2}[6,7]$.

A trigger that is highly selective against non prompt muons and has a high detection efficiency for leptonic decays of $\mathrm{W}^{ \pm}$and $\mathrm{Z}^{\circ}$ requires a transverse momentum threshold around $20 \mathrm{GeV} / \mathrm{c}$. In the detectors proposed for experiments at the LHC and the SSC a trigger with a sufficiently sharp threshold can be implemented with a number of planes segmented in strips of few $\mathrm{cm}$ width. The trigger rate, mainly due to semileptonic decays of charm and beauty particles will be of few $\mathrm{kHz}$ at $10^{34} \mathrm{~cm}^{-2} \mathrm{~s}^{-1}$ luminosity $[4,8]$.

Given the large area of the detectors and the high flux of particles, the rate due to accidental coincidences will be very large and it can be reduced to values smaller than the signal rate only with a high order of coincidence of detector planes with fine segmentation and very good time resolution.

Thus the requirements for detectors to be used in the first level muon trigger are:

- good uniformity of response over large areas;

- full efficiency and good time resolution at particle fluxes of $\approx 100 \mathrm{~Hz} / \mathrm{cm}^{2}$;

- easiness of segmentation;

- small sensitivity to low energy neutrons and photons;

- low noise rate. 
The results presented in ref. [1,2] were obtained with a RPC hodoscope of large dimensions and finely segmented in strips and can be summarized as follows:

- the time response is uniform over the surface;

- the efficiency is limited by the dead area in the gas volume due to the spacers that keep parallel the bakelite plates;

- plates with resistivity lower than $10^{11} \Omega \times \mathrm{cm}$ are better suited for operation at charged particle fluxes in excess of $10 \mathrm{~Hz} / \mathrm{cm}^{2}$;

- the efficiency and the time resolution deteriorate at particle fluxes larger than 30 $\mathrm{Hz} / \mathrm{cm}^{2}$;

- the noise rate, of few $\mathrm{kHz} / \mathrm{m}^{2}$, is mainly due to distortions of the electric field in the gas volume close to the spacers.

In order to improve the performance of the chambers we have built and installed in the RD5 experiment new planes of double-gap RPC and we have done a more systematic study of the behaviour of the efficiency and time resolution as a function of the particle flux.

\section{The double-gap Resistive Plate Chamber.}

A double-gap RPC consists of two separate chambers, built with the same technique described in detail in ref. [1], assembled as shown in Fig.1. The bakelite plates and the gas volumes are $2 \mathrm{~mm}$ thick. The average resistivity of the plates is the same as of the "low resistivity" RPC described in ref. [1]: $\rho \approx 410^{10} \Omega \times \mathrm{cm}$. The dimension of each chamber is $1 \times 2 \mathrm{~m}^{2}$. A single read-out plane, segmented in strips $2 \mathrm{~m}$ long and with $3.1 \mathrm{~cm}$ pitch, is placed between the two chambers to pick up the signal from both gas volumes. The outer face of the bakelite plates is coated with a graphite solution and the electric field is generated by applying a voltage difference between the two graphitecoated planes. Insulation of these planes is provided by a $300 \mu \mathrm{m}$ foil of PVC. The external planes of each chamber are connected to ground so that each strip acts as a transmission line. Each strip-line is terminated on $50 \Omega$ at both ends and one side is connected to the front-end electronics $[1,9]$.

The two chambers are staggered by $5 \mathrm{~cm}$ so that the spacers do not overlap. With such an arrangement separate gas volumes can be assembled together as shown in Fig.2 to form a large detector array without dead areas due to the spacers and the frames. In order to reduce the electric field in the gas volume close to the spacers, the graphite coating on the outer face of the bakelite plates has been removed from a small area of about $3 \mathrm{~cm}^{2}$ as shown in Fig.3. This is intended to reduce the noise rate of the RPC. The whole detector is made selfsustaining with a mechanical support consisting of two rigid plates $12 \mathrm{~mm}$ thick that are clamped together with two $U$ aluminum profiles as shown in Fig.4. Each plate is made of foam sandwiched between a thin internal layer of glass fiber and an external foil of aluminum that provides additional shielding. 
Eighteen such chambers, completely built and assembled by industry ", have been installed in the RD5 experiment.

\section{The RD5 experiment.}

The lay-out of the RD5 experiment has been described in detail elsewhere [10]. It consists of a 10 interaction lengths sampling calorimeter [11], made of stainless steel plates alternated with proportional gas tubes, immersed in a $3 \mathrm{~T}$ magnetic field produced by a superconducting coil, followed by a toroidal magnet made of four iron walls. The thickness of the walls normal to the beam direction is $1.8 \mathrm{~m}$ and the field in the toroid is 1.5 $\mathrm{T}$. There are three muon measurement stations: the first placed between the calorimeter and the toroid, the second between the iron walls and the third behind the toroid. Each station comprises large area drift chambers [12] and RPC planes [1].

An array of ten double-gap RPC is placed in front of the experiment to veto the offtime halo particles accompanying the beam. The array, of dimensions $4 \times 4.5 \mathrm{~m}^{2}$, has a rectangular hole of variable size in the centre to allow the beam to pass through. An efficient veto over a large area is needed for the study of the punchthrough of hadron showers at various depths in the detector [10].

The other eight double-gap RPC have been assembled together to form four detector planes of $2 \times 2 \mathrm{~m}^{2}$ surface. These detectors (hereafter called Y9, Y10, Y11 and Y12) were placed in the second and in the third muon measurement station with one plane on either side of the drift chambers. The 64 strips of each detector measure the coordinate in the bending plane and were read out in the RD5 data acquisition. Each plane provided a Fast-OR signal that could be used in the trigger of the experiment.

\section{Performance of the double-gap RPC.}

The data presented here refer to the latter four double-gap RPC planes and were taken in spring 1993 with a muon beam of $200 \mathrm{GeV} / \mathrm{c}$ momentum. The beam was defined by the coincidence of two scintillators of dimensions $10 \times 10 \mathrm{~cm}^{2}$ placed upstream of the calorimeter. Thus, before reaching the RPC planes in the two measurement stations, the muons traverse 3.5 and $5.3 \mathrm{~m}$ of iron respectively. The profile of the muon beam was measured with multiwire chambers of $2 \mathrm{~mm}$ spacing before the calorimeter and with the drift chambers at the position of the RPC planes.

The data were taken with a gas mixture of $58 \%$ argon, $38 \%$ butane and $3.8 \%$ freon. Fig. 5 shows, as a function of the supply voltage, the efficiency of each plane, measured by the coincidence of the Fast-OR with the scintillators trigger, and the rate of the Fast-OR, measured when there was no beam. The average rate per unit surface is 7 $\mathrm{kHz} / \mathrm{m}^{2}$ : this represents an improvement of more than a factor two per single RPC with

* General Technica, I-03030 Colli, Italy. 
respect to previous measurements done with single-gap RPC built with plates of similar resistivity and operated in the same conditions [1]. The efficiency data refer to a muon flux of $\approx 10 \mathrm{~Hz} / \mathrm{cm}^{2}:$ the chambers reach an efficiency of $99 \%$ or higher. The measurements were done supplying the two gas volumes with a common high voltage line. We have also measured the efficiency curves powering one gas volume at the time and we found that the operation voltages were equal for chambers Y10 and Y12, but rather different for chambers $\mathrm{Y} 9$ and $\mathrm{Y} 11$. Thus we expect slightly poorer performance for these two chambers.

Measurements of the efficiency and of the time resolution as a function of the beam flux were done with the high voltage set at 500-1000 V above the beginning of the efficiency plateau: the operation voltages, shown in Fig.5, were chosen to study different working conditions. The local flux traversing the RPC was defined by the rate of the trigger scintillators and the beam profile measured with the drift chambers at the positions of the RPC. The efficiency is quoted for an area of $4 \times 4 \mathrm{~cm}^{2}$ that insures a uniform distribution of the particles traversing the detector planes. Thus the data refer to only a small surface of the whole RPC. Fig.6a shows the efficiency of the four double-gap RPC as a function of the flux. The same results are shown in Fig. $6 \mathrm{~b}$ for the single-gap RPC already described in ref. [1]. The only difference with respect to the previous data, taken during a run dedicated to the measurement of the punchthrough of hadron showers [10], is that the operation voltage of the single-gap RPC was increased by about $400 \mathrm{~V}$.

In Fig.7 we plot the efficiency measured in different rings of the detector planes centred on the maximum of the particle flux. The result shows that the reduction of the efficiency in the centre has little effect on the efficiency of the detector in the adjacent regions: the reduction of the electric field in the gas volume is localized in the region where the particle flux is larger. For single-gap RPC the-local efficiency is limited by the spacers in the gas volume that are placed at a distance of $10 \mathrm{~cm}$ from one another.

From these results we can conclude that RPC built with phenolic plates of low resistivity, $\rho \approx 410^{10} \Omega \times \mathrm{cm}$, have a satisfactory behaviour up to local particle fluxes of $\approx 50 \mathrm{~Hz} / \mathrm{cm}^{2}$, while double-gap RPC can operate safely at local particle fluxes of $\approx 100$ $\mathrm{Hz} / \mathrm{cm}^{2}$. It should be noted, however, that all measurements were done with a small surface exposed to the beam flux. When exposing the whole chamber to an uniform flux, a larger reduction of the efficiency has been observed [13] that has been interpreted as due to the voltage drop over the electrodes caused by the current flow needed to recharge the plates. A large improvement of the rate performance of RPC can be obtained by operating the chambers at a lower gain [14]. In this case the requirements on the gain and bandwidth of the front-end electronics exceed the performance of that used during the measurements presented here. 


\section{Space resolution.}

We have shown that in single-gap RPC the discharge is localized in a region of a few $\mathrm{mm}$ [15] and that the space resolution obtained with a strip read-out pitch of $3 \mathrm{~cm}$ is indeed compatible with the charge distribution induced by a localized discharge. In double-gap RPC, however, the fluctuations in the discharge in the two separate gas volumes contribute to broaden the charge distribution. Since both detectors are read out with the same electronics, the "effective threshold" of double-gap RPC is half than for single-gap RPC. We then expect that the localization performance of double-gap RPC is somehow poreer than for single-gap RPC.

To study the space resolution of the detector, we define clusters made with adjacent strips and we compare the position of the centre of the cluster with that of the track reconstructed in the drift chambers. Fig. 8 shows the distribution of the position of the track in the direction orthogonal to the strips, separately for clusters made with one and two strips: the probability of two-strip clusters is large close to the boundary between two strips and for double-gap RPC extends over a wider interval.

Fig.9a shows the distribution of the distance of the centre of the cluster from the position of the track. For double-gap RPC the r.m.s. width is $7 \mathrm{~mm}$, similar to that of single-gap RPC [1]. A relevant figure for a tracking trigger is the size of the clusters: this is given in Fig $9 \mathrm{~b}$ that shows the distribution of the distance of the centre of any strip of a cluster from the position of the track. The FWHM of the distribution is $4.2 \mathrm{~cm}$ for double-gap RPC, the same distribution for single-gap RPC has a FWHM of $3.7 \mathrm{~cm}$.

Double-gap RPC, that operate with a lower effective threshold, show a broadening of the clusters and we expect that, operating the RPC in these conditions, there will be little gain in space resolution by reducing the size of the strips. A smaller extension of the discharge can be obtained operating the RPC at a lower gas gain, either reducing the electric field or using a more quenching gas mixture [14]. We stress however that the results obtained in the present conditions are fully adequate for most of the muon trigger schemes presented in the proposed experiments [4].

\section{Time resolution.}

The time response of the four double-gap RPC planes has been studied by measuring the delay between the signal of the trigger hodoscope and those of the Fast-OR of each plane. We noticed a small systematic difference in the time response of the single strips, but no attempt was done to correct for this effect, neither to correct for the jitter of the trigger signal. On the basis of the four independent measurements, we estimate a r.m.s. contribution of $0.9 \mathrm{~ns}$ from the trigger.

Fig.10a shows the distribution of the delay for particle fluxes of 25, 100 and 300 $\mathrm{Hz} / \mathrm{cm}^{2}$. With increasing particle flux there is an increase of the average value, due to the slower response of the RPC at smaller values of the local electric field, and a broadening 
of the distribution. We define delay the average value of the distribution, and resolution the r.m.s. width of a gaussian fit to the distribution. Referring to the data of Fig.10a (chamber Y11), the time distribution at $100 \mathrm{~Hz} / \mathrm{cm}^{2}$ has a small non gaussian tail, the resolution is $2.7 \mathrm{~ns}$, and the time interval that contains $99 \%$ of the events is $20 \mathrm{~ns}$. The distribution of the time difference between the two RPC in the third measurement station (chambers Y11 and Y12) is shown in Fig.10b. The fraction of events with a coincidence within $25 \mathrm{~ns}$ is $1.00,0.98,0.92$ for fluxes of 25,100 and $300 \mathrm{~Hz} / \mathrm{cm}^{2}$ respectively.

Fig. 11 shows the delay and the time resolution as a function of the particle flux for the four planes. Chamber $\mathrm{Y} 9$ has the longest tail in the delay distribution, for the reason explained in Section 4. The best results, both in efficiency and time resolution, are obtained with chamber Y10 that has an operation voltage of about $1000 \mathrm{~V}$ above the beginning of the efficiency plateau.

\section{Conclusion.}

We have built and installed in the RD5 experiment at CERN $36 \mathrm{~m}^{2}$ of double-gap Resistive Plate Chambers with the read-out plane segmented in strips of $3 \mathrm{~cm}$ pitch. The efficiency, the localization properties and the time response have been studied as a function of the particle flux with a muon beam of several $\mathrm{cm}^{2}$ section. For a local particle flux of $100 \mathrm{~Hz} / \mathrm{cm}^{2}$ the double-gap RPC operated in discharge mode have good efficiency and a time resolution of $2-3 \mathrm{~ns}$, though the space resolution is slightly worse than that of single-gap RPC.

Tests done with small RPC built with the same material and with the same assembly technique, but operated at a lower gas gain and read out with a larger gainxbandwidth front-end electronics, have shown that the efficiency and timing performance are largely improved at large particle flux. Provided these results are confirmed with large scale RPC and with a variety of safe gas mixtures, these detectors seem adequate to build a fast tracking muon trigger in the central region of experiments at the LHC able to provide a sufficiently sharp selection in transverse momentum within a delay of few $100 \mathrm{~ns}$ and to identify the interaction bunch crossing directly at level one.

\section{Acknowledgements}

We would like to thank F.Cidronelli, S.Di Marco, L.Distante, E.Reali, A.Rossi and E.Tusi for the assembly and test of the read-out electronics and for their help during the installation of the detectors. We thank warmly all our colleagues of the RD5 collaboration for their help and advice during the installation and the data taking of the experiment. 


\section{References}

[1] M.Andlinger et al.(RD5 Collaboration): "Study of Resistive Plate Chambers for muon detection at hadron colliders", Internal Note 1015, Dipartimento di Fisica Università di Roma La Sapienza, 9.7.1993, to be published in Nuclear Instruments and Methods in Physics Research.

[2] L.Pontecorvo (RD5 Collaboration): Proceedings of the International Workshop on Resistive Plate Chambers in Particle Physics and Astrophysics, ed. S.Ratti, G.Ciapetti and R.Santonico, Scientifica Acta 8 (1993) 145.

[3] M.Della Negra et al.: "Study of muon triggers and momentum reconstruction in a strong magnetic field for a muon detector at LHC", CERN/DRDC/90-36, DRDC/P7, 1.9.1990.

[4] CMS, Letter of Intent, CERN/LHCC/92-3, 1.10.1992; ATLAS, Letter of Intent, CERN/LHCC/92-4, 1.10.1992; L3P, Letter of Intent, CERN/LHCC/92-5, 1.10 .1992 .

[5] F.Ceradini et al.: Proceedings of the Large Hadron Collider Workshop, CERN/9010, ECFA/90-133, eds. G.Jarlskog and D.Rein, vol.III, page 99; R.Santonico: Proceedings of the Large Hadron Collider Workshop, CERN/90-10, ECFA/90133, eds. G.Jarlskog and D.Rein, vol.III, page 838; C.Bacci et al.: Nuclear Instruments and Methods in Physics Research, A315 (1992) 102.

[6] A.Ferrari: private communication.

[7] I.Pless: Proceedings of the International Workshop on Resistive Plate Chambers in Particle Physics and Astrophysics, ed. S.Ratti, G.Ciapetti and R.Santonico, Scientifica Acta 8 (1993).

[8] A.Nisati: Proceedings of the International Workshop on Resistive Plate Chambers in Particle Physics and Astrophysics, ed. S.Ratti, G.Ciapetti and R.Santonico, Scientifica Acta 8 (1993) 61.

[9] R.Cardarelli: "Read-out system for Resistive Plate Chambers", presented at the LXXIII Congresso Nazionale della Società Italiana di Fisica, Neaples, Italy,
13.10.1987.

[10] M.Aalste et al.(RD5 Collaboration): Zeitschrift für Physik C60 (1993) 1.

[11] F.Bakker et al.: Nuclear Instruments and Methods in Physics Research, A330 (1993) 44.

[12] K.Eggert et al.: Nuclear Instruments and Methods, 176 (1980) 217.

[13] M.Iori and F.Massa: Nuclear Instruments and Methods in Physics Research, A306 (1991) 159; I.Crotty et al.: Nuclear Instruments and Methods in Physics Research, A329 (1993) 133.

[14] R.Cardarelli, A.DiCiaccio and R.Santonico: Nuclear Instruments and Methods in Physics Research, A333 (1993) 39; I.Crotty et al.: "The non spark mode and high rate operation of Resistive Plate Chambers", CERN-PPE/93-180, 13.9.1993, submitted to Nuclear Instruments and Methods in Physics Research.

[15] F.Ceradini et al.: "Measurement of the hit distribution in Resistive Plate Chambers equipped with a digital read-out of the charge induced on strips", Internal Note 990, Dipartimento di Fisica Università di Roma La Sapienza, 9.12.1991. 


\section{Figure Captions}

Fig.1 Cross section of a double-gap RPC.

Fig. 2 Detector array made of double-gap RPC.

Fig.3 Assembly of the bakelite plates.

Fig. 4 Assembly of a double-gap RPC.

Fig.5 Efficiency (squares - left scale) and rate per unit surface (circles - right scale) as a function of the supply voltage.

Fig.6 Efficiency as a function of the particle flux for (a) double-gap RPC and (b) single-gap RPC.

Fig.7 Efficiency as a function of the distance from the beam centre for (a) double-gap RPC and (b) single-gap RPC.

Fig.8 Distribution of the position of the track in the direction orthogonal to the strips for clusters made of one and two strips: (a) double-gap RPC, (b) single-gap RPC.

Fig.9 Distribution of the distance of the position of the track from (a) the centre of the cluster and (b) the centre of any strip in the cluster.

Fig.10 Distribution of the delay (a) relative to the trigger and (b) between two doublegap RPC for different particle fluxes: $25 \mathrm{~Hz} / \mathrm{cm}^{2}$ full line, $100 \mathrm{~Hz} / \mathrm{cm}^{2}$ dashed line, $300 \mathrm{~Hz} / \mathrm{cm}^{2}$ dotted line.

Fig.11 Delay (a) and time resolution (b) as a function of the particle flux. 


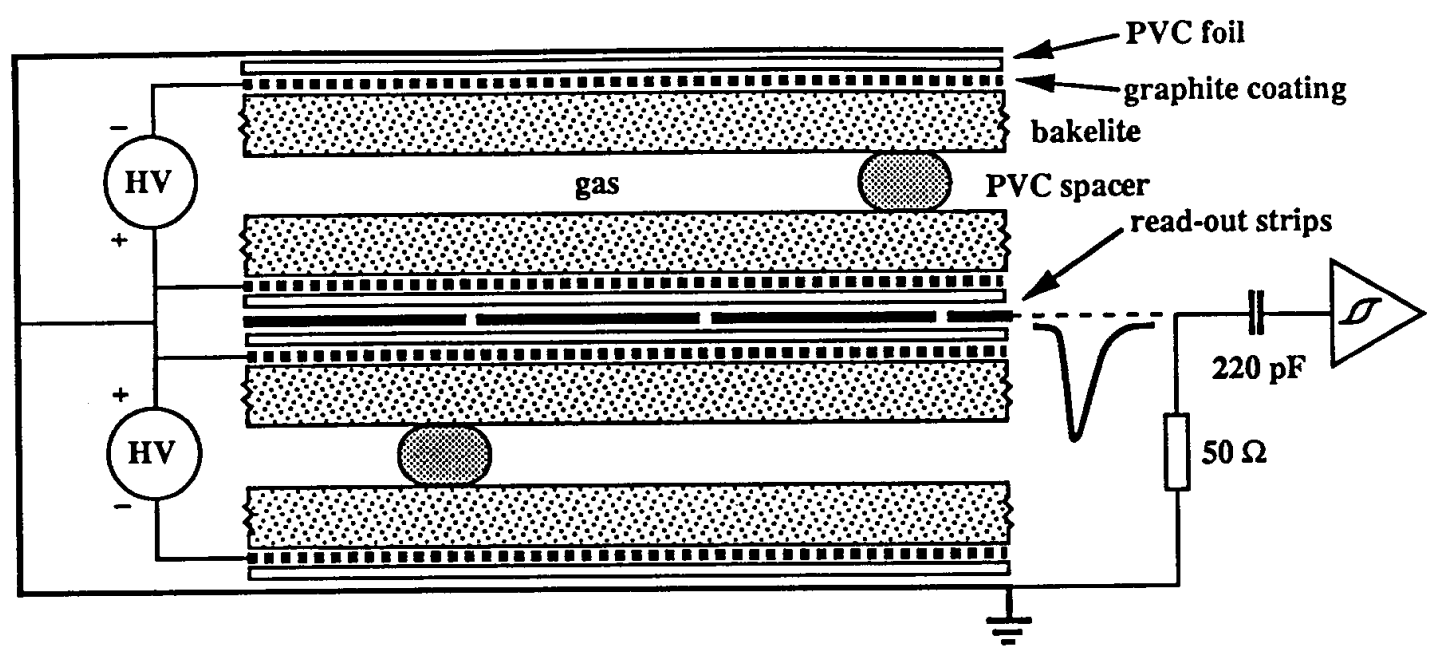

Fig.1

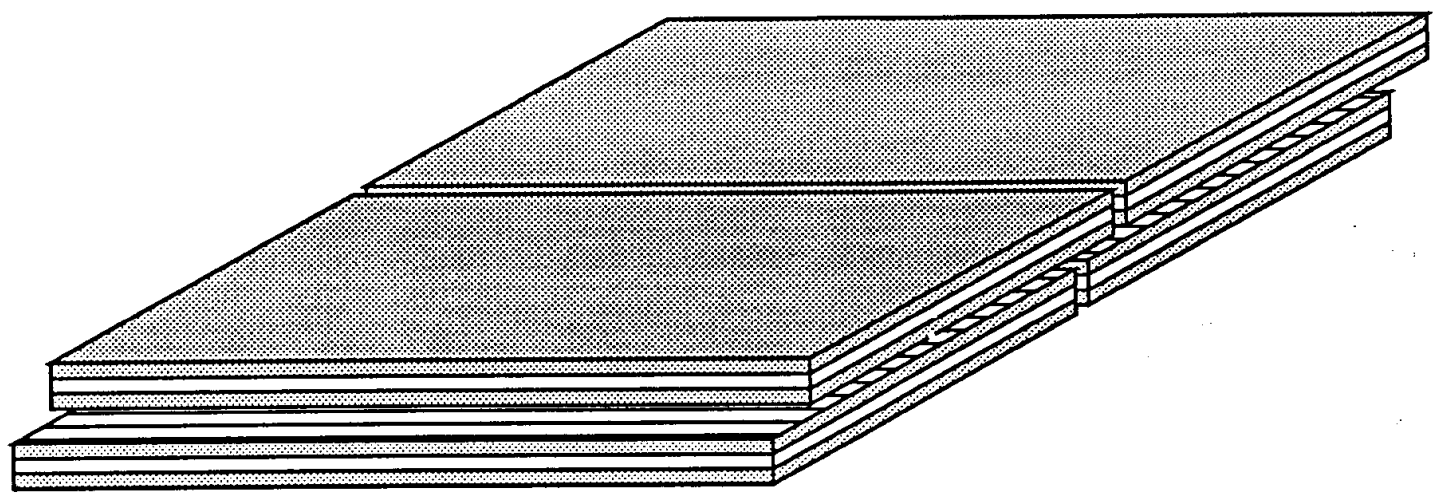

Fig.2 


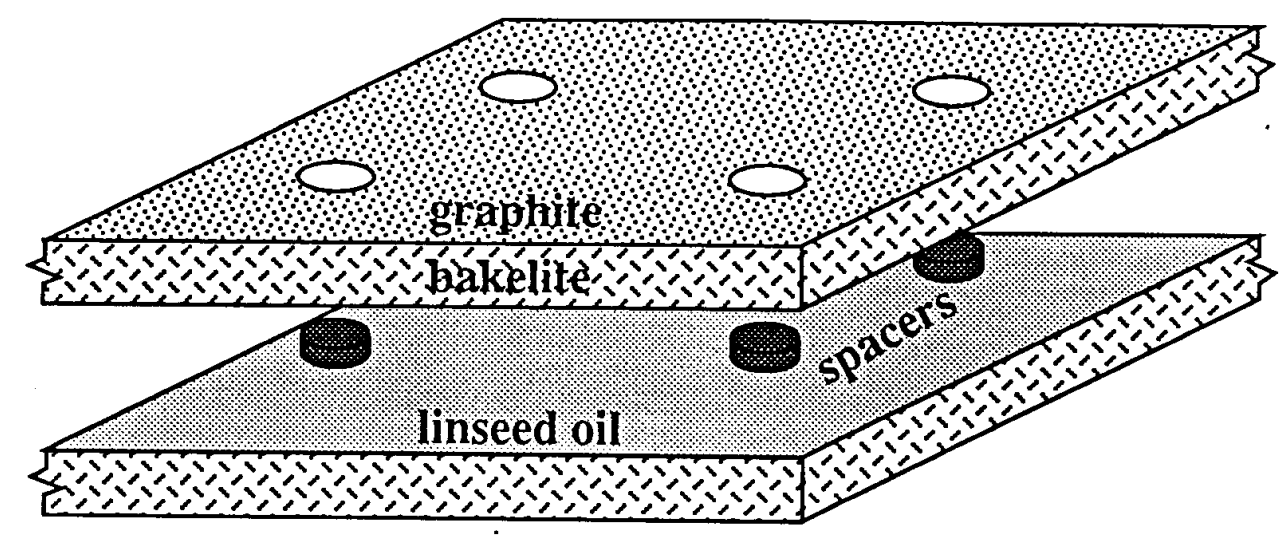

Fig.3

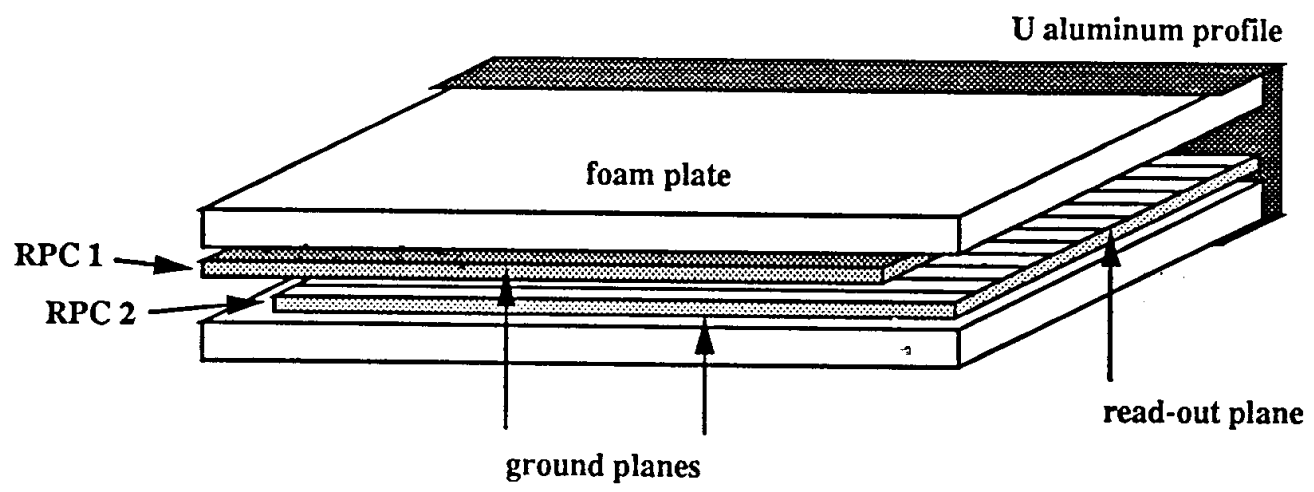

Fig.4 

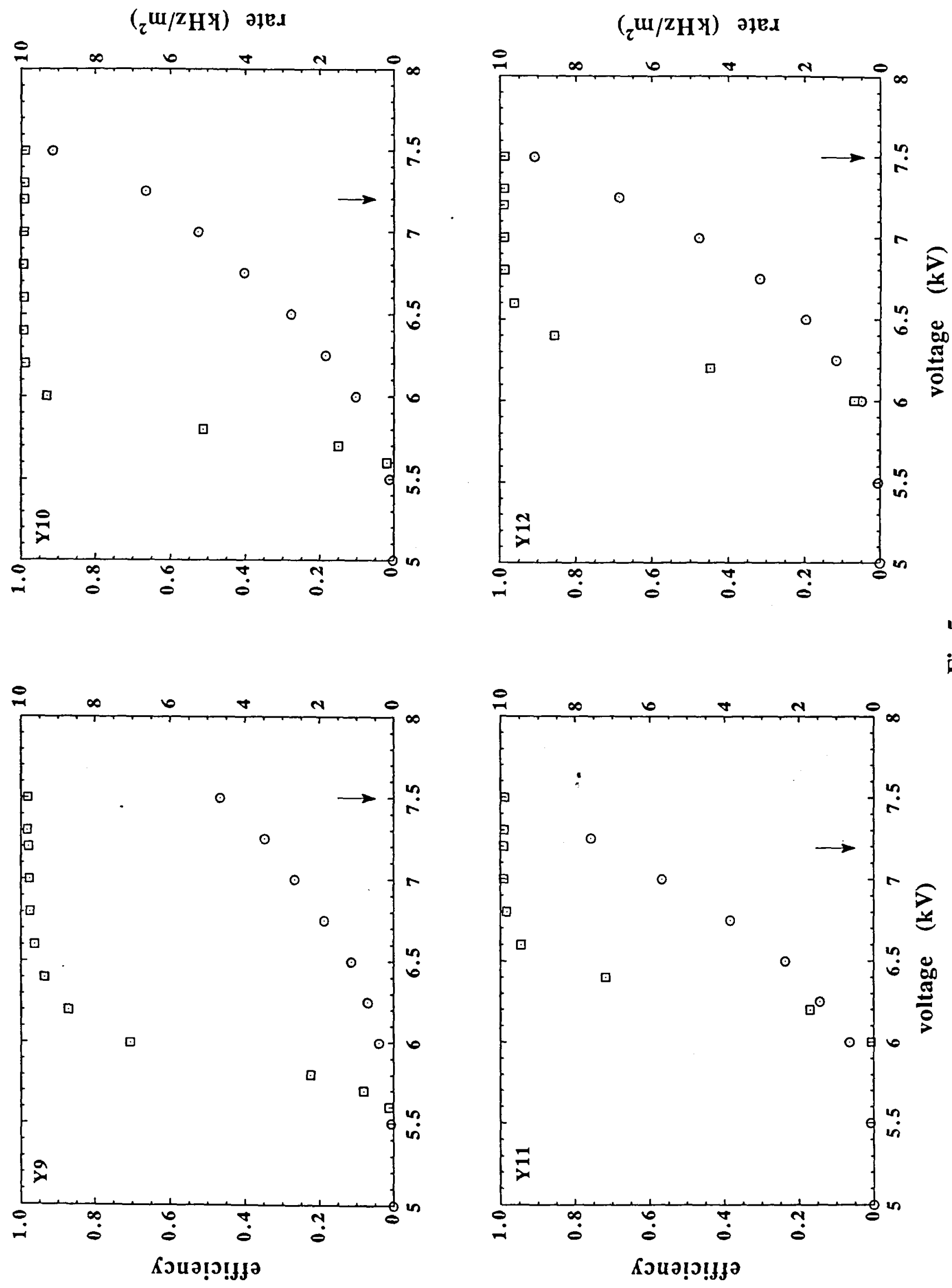

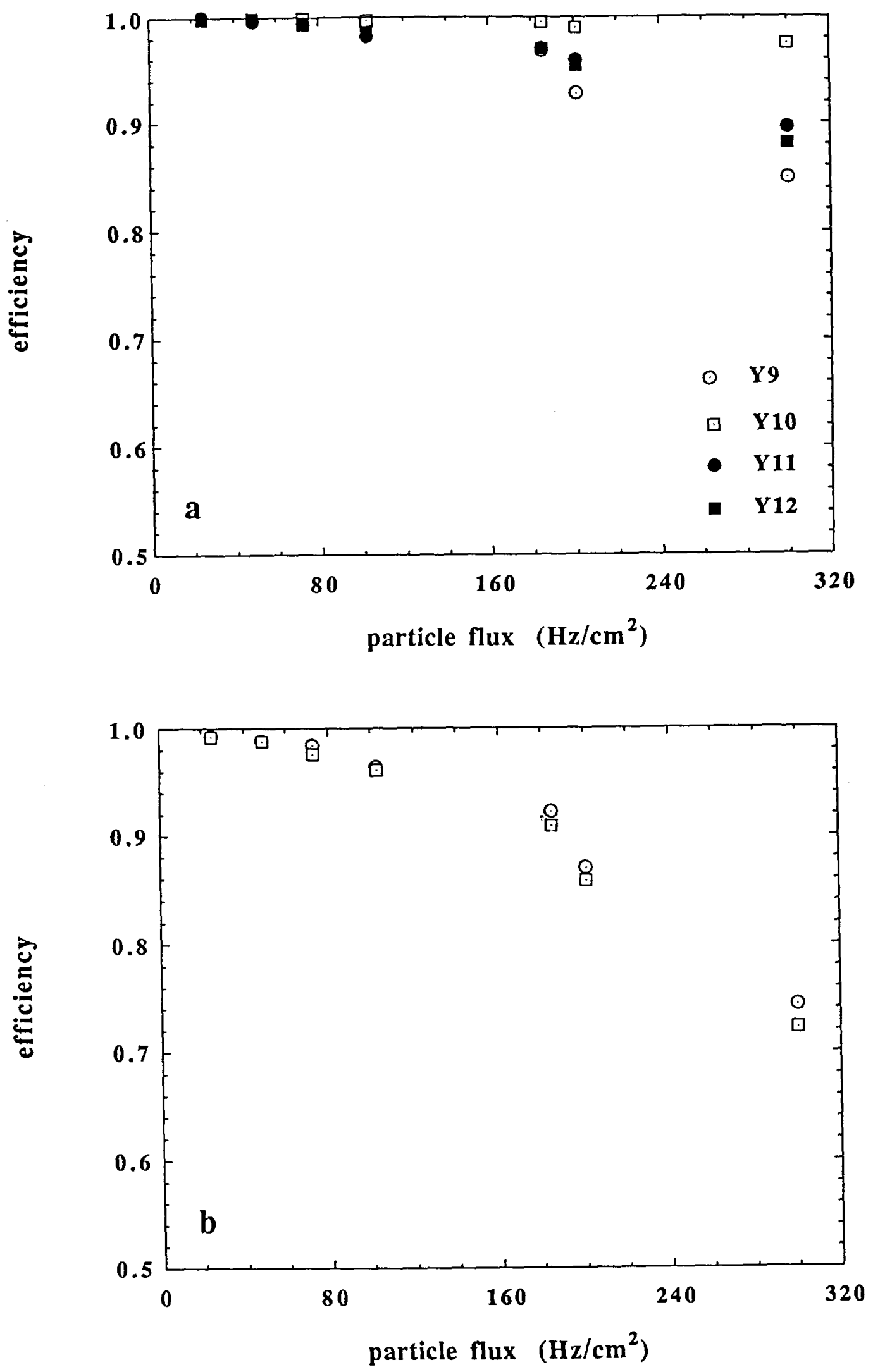

Fig.6 

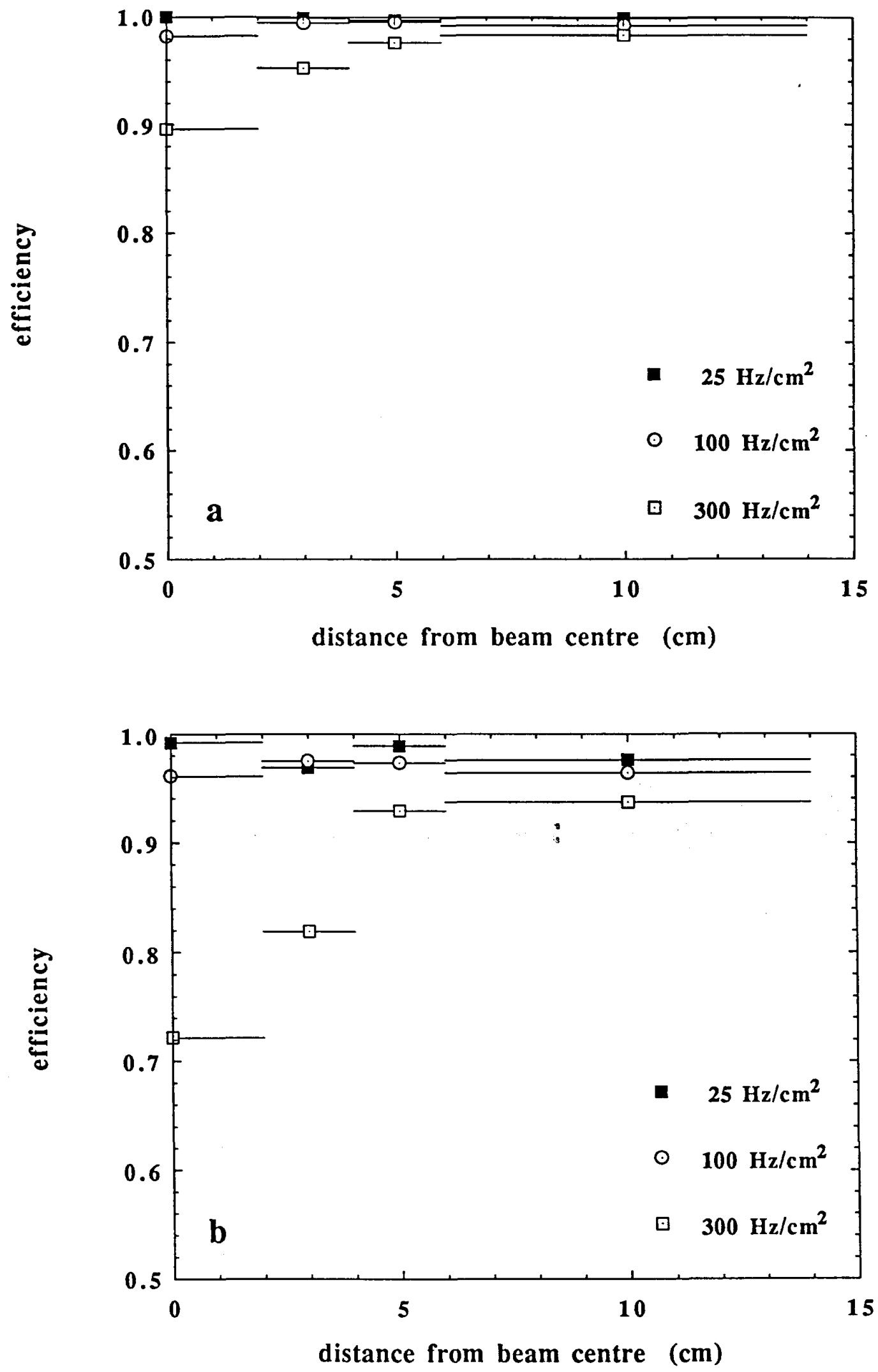

Fig.7 

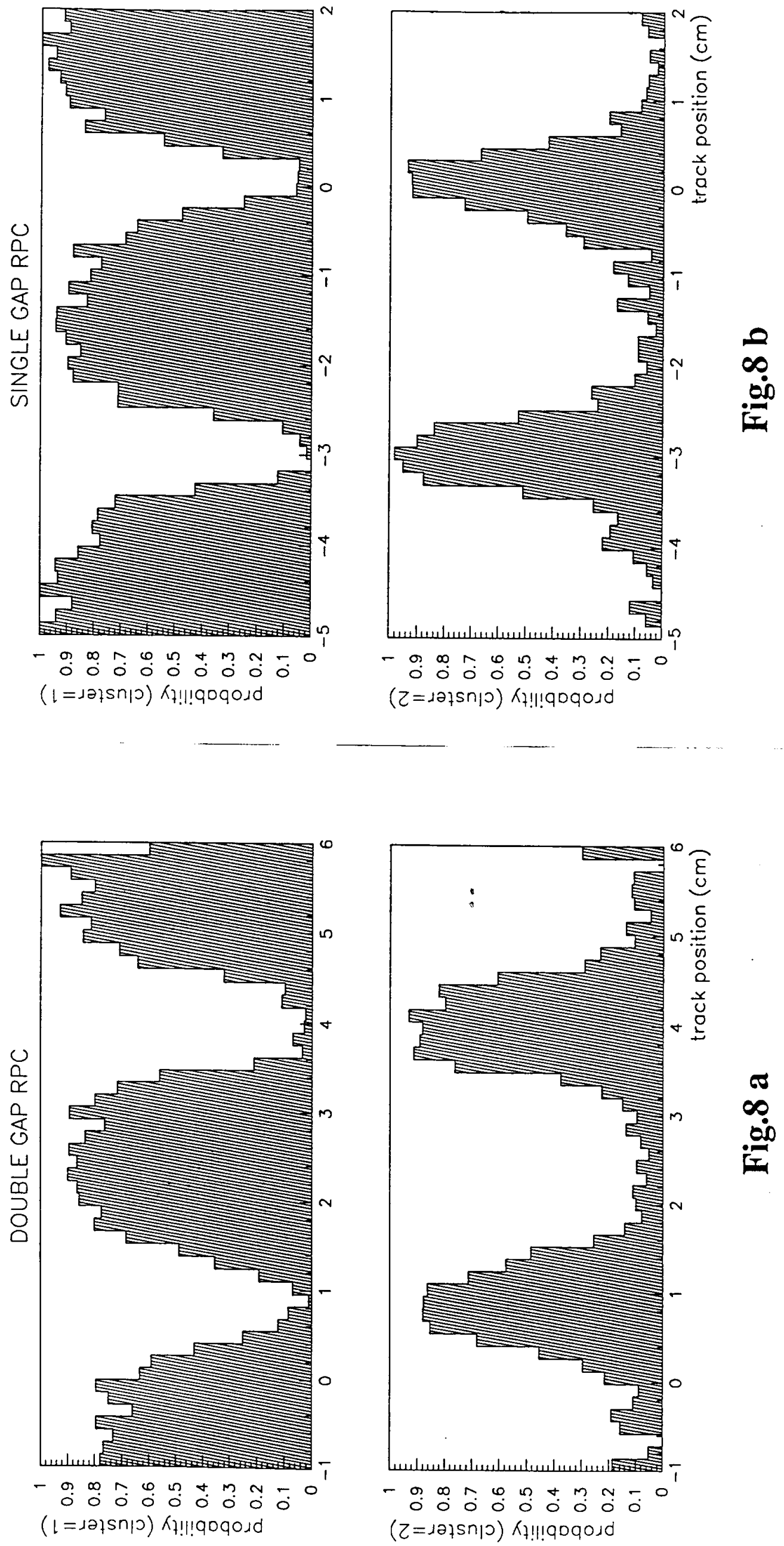

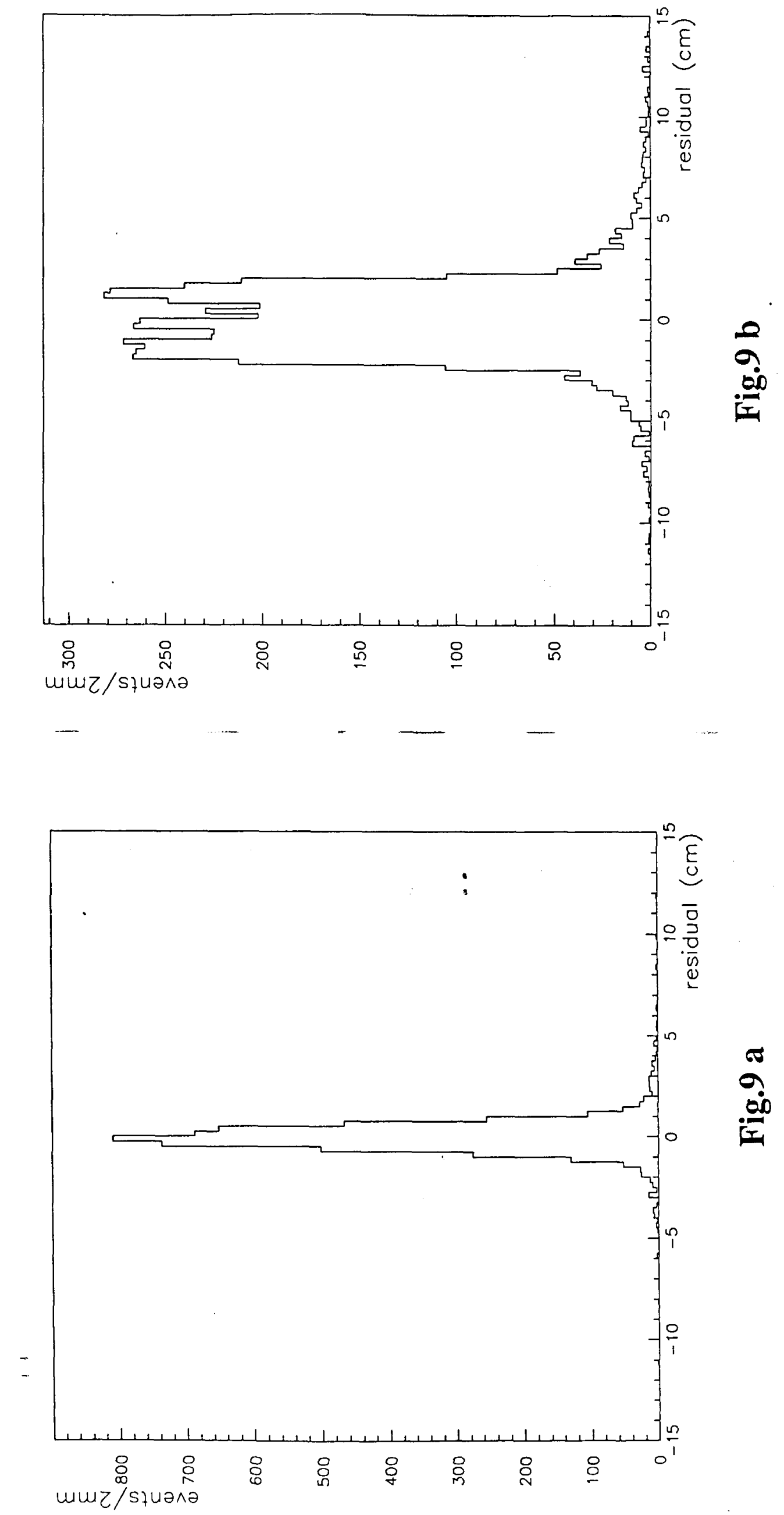

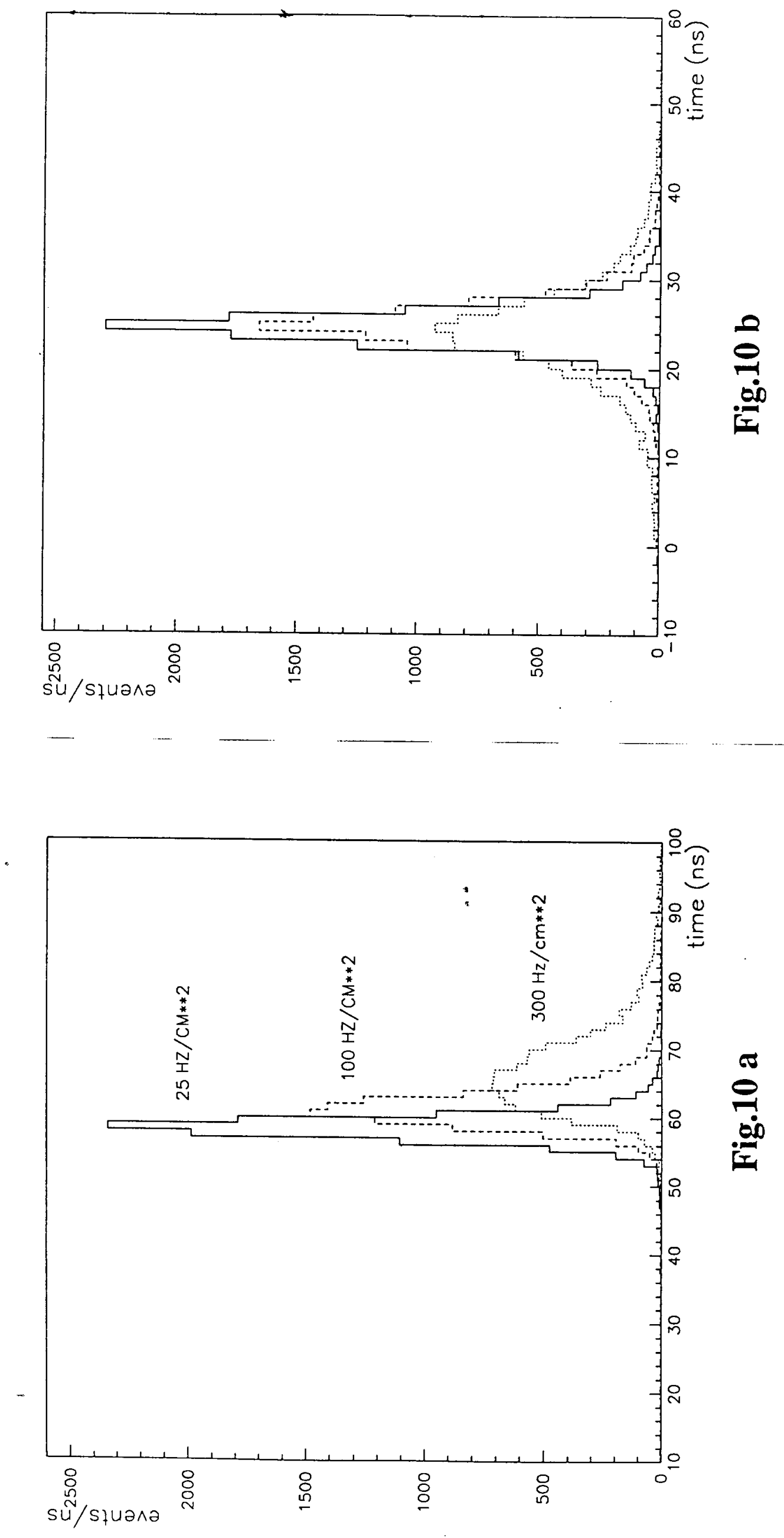

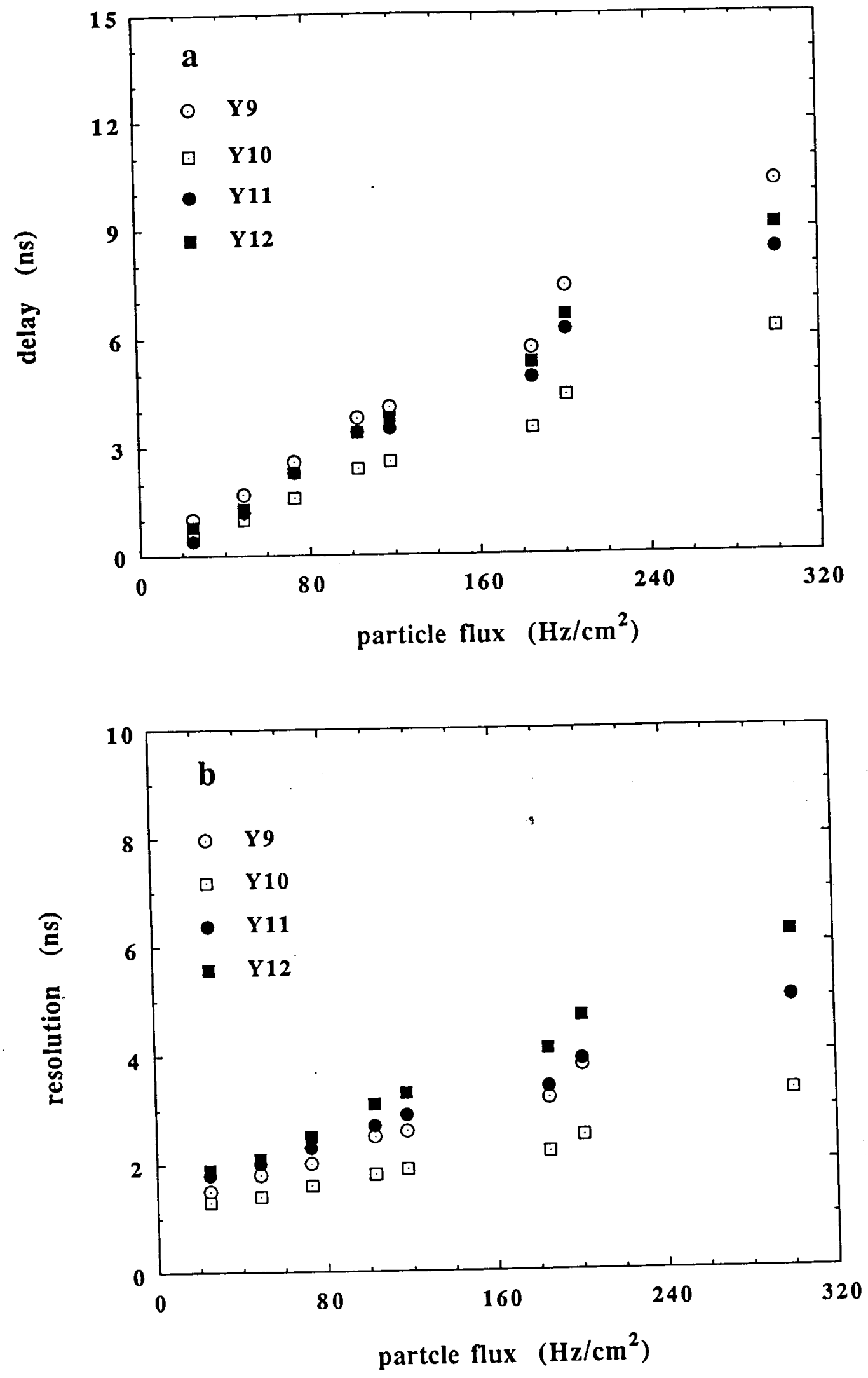

Fig.11 
$n$ 